\title{
BFKL equation with running QCD coupling and HERA data
}

\author{
Eugene Levin $^{a, b}$ and Irina Potashnikova ${ }^{b}$ \\ ${ }^{a}$ Department of Particle Physics, School of Physics and Astronomy, \\ Tel Aviv University, Tel Aviv, 69978, Israel \\ ${ }^{b}$ Departamento de Fúsica, Universidad Técnica Federico Santa María, \\ and Centro Científico-Tecnológico de Valparaíso, \\ Casilla 110-V, Valparaiso, Chile \\ E-mail: leving@post.tau.ac.il, irina.potashnikova@usm.cl
}

ABSTRACT: In this paper we developed approach based on the BFKL evolution in $\ln \left(Q^{2}\right)$. We show that the simplest diffusion approximation with running QCD coupling is able to describe the HERA experimental data on the deep inelastic structure function with good $\chi^{2} /$ d.o.f. $\approx 1.3$. From our description of the experimental data we learned several lessons; (i) the non-perturbative physics at long distances started to show up at $Q^{2}=0.25 \mathrm{GeV}^{2}$; (ii) the scattering amplitude at $Q^{2}=0.25 \mathrm{GeV}^{2}$ cannot be written as sum of soft Pomeron and the secondary Reggeon but the Pomeron interactions should be taken into account; (iii) the Pomeron interactions can be reduced to the enhanced diagrams and, therefore, we do not see any needs for the shadowing corrections at HERA energies; and (iv) we demonstrated that the shadowing correction could be sizable at higher than HERA energies without any contradiction with our initial conditions.

KeYwords: Deep Inelastic Scattering (Phenomenology), QCD Phenomenology

ARXIV EPRINT: 1307.7823 


\section{Contents}

1 Introduction 1

2 BFKL equation with running $\alpha_{S}$ as the evolution equation 2

2.1 The equation 2

2.2 Green function and the set of Pomerons 3

$\begin{array}{ll}2.3 \text { Main formulae } & 7\end{array}$

3 Description of the HERA data $\quad 8$

4 Conclusions 13

\section{Introduction}

High energy (low $x$ ) deep inelastic scattering (DIS) probes the gluon density in the hadron. Its energy evolution is determined by the BFKL equation [1-4] which sums the leading log terms of the order of $\left(\alpha_{S} \ln (1 / x)\right)^{n}$. During the past two decades different facets of the BFKL dynamics have been discussed in the number of papers (see Res. [3-5] for reviews). In our opinion, such close attention to the BFKL dynamics is rooted in two causes. First, the increase of the gluons density at high energy $\left(\propto(1 / x)^{\lambda}\right)$ has been observed experimentally at HERA [6]; and we need to take into account the BFKL dynamics to understand this increase. In other words, we can view on the BFKL dynamics as the evolution [7] of the gluon density at low Bjorken $x$ in DIS. However, the BFKL equation gives an inspiration or might be even the educated guess for the non-perturbative origin of the soft Pomeron contribution or, in general, it can create ideas about the high energy asymptotical behaviour of the scattering amplitude in the confinement region. We have even indication that BFKL equation generates the infinite number of Pomerons (Regge poles) for the running QCD coupling (see refs. $[3,4,7,8]$ ).

Recently, in the interesting papers (see refs. [9-12]) the consistent approach, based on the point of view that the BFKL equation is the theory of the reggeons, has been developed and applied to description of the HERA data on DIS. The successful representation of the data undermine the widespread prejudice that the BFKL evolution is not needed for a description of the HERA data (see refs. [13-20]), In this paper we are going to hammer the last nail in the coffin of this prejudice showing that the good fit of HERA data is naturally appeared in the evolution equation approach to the BFKL dynamics. 


\section{BFKL equation with running $\alpha_{S}$ as the evolution equation}

\subsection{The equation}

The NLO BFKL equation can be written in the form (see $[9,10,21,22])$

$$
\begin{aligned}
\frac{\partial N\left(k_{\perp}, Y\right)}{\partial Y}=\bar{\alpha}_{S}\left(k_{\perp}\right) \int d^{2} & k_{\perp}^{\prime} K_{L O}\left(k_{\perp}, k_{\perp}^{\prime}\right) N\left(k_{\perp}^{\prime}, Y\right) \\
& +\bar{\alpha}_{S}^{2}\left(k_{\perp}\right) \int d^{2} k_{\perp}^{\prime} K_{N L O}\left(k_{\perp}, k_{\perp}^{\prime}\right) N\left(k_{\perp}^{\prime}, Y\right)
\end{aligned}
$$

where

$$
N\left(k_{\perp}, Y\right)=\frac{1}{\sqrt{\bar{\alpha}_{S}\left(k_{\perp}\right)}} \int d^{2} x e^{i \vec{k}_{\perp} \cdot \vec{x}} \int d^{2} b \frac{N(x, b ; Y)}{x^{2}}
$$

with $N(r, b: Y)$ being the imaginary part of the scattering amplitude of the dipole with size $x$.

$$
\bar{\alpha}_{S}\left(k_{\perp}\right)=\left(N_{c} / \pi\right) \alpha_{S}\left(k_{\perp}\right)=\frac{1}{b \ln \left(k_{\perp}^{2} / \Lambda_{\mathrm{QCD}}^{2}\right)}
$$

and

$$
K_{L O}\left(k_{\perp}, k_{\perp}^{\prime}\right)=\frac{1}{\left(\vec{k}_{\perp}-\vec{k}_{\perp}^{\prime}\right)^{2}}-\frac{k_{\perp}^{2}}{\left(\vec{k}_{\perp}-\vec{k}_{\perp}^{\prime}\right)^{2}\left(\left(\vec{k}_{\perp}-\vec{k}_{\perp}^{\prime}\right)^{2}+k_{\perp}^{\prime 2}\right)} \delta^{(2)}\left(\vec{k}_{\perp}-\vec{k}_{\perp}^{\prime}\right)
$$

while $K_{N L O}\left(k_{\perp}, k_{\perp}^{\prime}\right)$ is written in ref. [21, 22].

One can see that in eq. (2.1) we do not use the triumvirate structure [24-27] of the LO BFKL for running $\bar{\alpha}_{S}$ which looks as follows:

$$
\frac{\partial N\left(k_{\perp}, Y\right)}{\partial Y}=\int d^{2} k_{\perp}^{\prime}\left(\frac{\bar{\alpha}_{S}\left(k_{\perp}^{\prime}\right) \bar{\alpha}_{S}\left(\vec{k}_{\perp}-\vec{k}_{\perp}^{\prime}\right)}{\bar{\alpha}_{S}\left(k_{\perp}\right)}\right) K_{L O}\left(k_{\perp}, k_{\perp}^{\prime}\right) N\left(k_{\perp}^{\prime}, Y\right)
$$

The advantage of this expression that it preserves the bootstrap equations for the reggeized gluon that has been proven in the NLO BFKL approach [23]. On the other hand eq. (2.5) takes into account part of the NLO corrections of eq. (2.1) which are not the largest contribution to $K_{N L O}$ in eq. (2.1). Since the main goal of this paper to clarify some rather qualitative features of the BFKL dynamics with running QCD coupling we feel it is reasonable to use the LO contribution to the simple equation (see eq. (2.1)) following the example of refs. [9-12].

Finally, in this paper we are going to discuss the following equation:

$$
\frac{\partial N\left(k_{\perp}, Y\right)}{\partial Y}=\bar{\alpha}_{S}\left(k_{\perp}\right) \int d^{2} k_{\perp}^{\prime} K_{L O}\left(k_{\perp}, k_{\perp}^{\prime}\right) N\left(k_{\perp}^{\prime}, Y\right)
$$

with $\bar{\alpha}_{S}\left(k_{\perp}\right)$ and $K_{L O}$ are given by eq. (2.3) and eq. (2.4), respectively. 


\subsection{Green function and the set of Pomerons}

The BFKL equation (see eq. (2.1)) is the typical evolution equation in $Y$ for solving which we need to fix the initial condition, $N\left(k_{\perp}, Y=Y_{0}\right)$, at any values of $k_{\perp}$. Having this initial condition we can find $N\left(k_{\perp}, Y\right)$ at any value of $Y$. However, such way of solution is based on knowledge of $N\left(k_{\perp}, Y=Y_{0}\right)$ in the confinement region $k_{\perp} \leq \Lambda_{\mathrm{QCD}}$. Since we have no theory of the confinement, it suffers theoretically uncontrollable assumption on the behaviour of the initial condition at small transverse momentum. However, the situation is even worse: due to diffusion in $\ln \left(k_{\perp} / \Lambda_{\mathrm{QCD}}\right)$ the BFKL evolution gives small transverse momenta at large $Y$ even from the sufficiently large transverse momenta in the initial condition [28] (see figure 3). We suggest to look at the BFKL equation in a different way to avoid the above difficulties. In this paper, we solve the BFKL equation in a way which is typical for the solution of the DGLAP evolution equation. We fix the boundary conditions at fixed $k_{0, \perp}\left(r_{0}=\ln \left(k_{0, \perp}^{2} / \Lambda_{\mathrm{QCD}}^{2}\right)\right)$ using the phenomenological function $N\left(k_{0, \perp}, Y\right)$. Using this boundary condition we find $N\left(k_{\perp}, Y\right)$ at any value of $k_{\perp}$. In this way of solution we include the entire information about confinement in the boundary condition. The advantage of such a way of finding solution that we can use the Pomeron phenomenology to fix the boundary condition and we will not face other confinement problems in the framework of this solution.

In our approach treating the BFKL equation as evolution in $k_{\perp}$ we need to find a Green function $\left.\left(G_{(} Y, r\right)\right)$ which satisfies the following initial condition:

$$
\left.G_{(} Y-Y_{0}, r=r_{0}\right)=\delta\left(Y-Y_{0}\right) \text { where } r \equiv \ln \left(k_{\perp}^{2} / \Lambda_{\mathrm{QCD}}^{2}\right)
$$

Using this function we can find the solution to the BFKL evolution equation $(N(r, Y))$ with given initial gluon distribution $N_{\text {in }}\left(Y_{0}, r=r_{0}\right)$

$$
N_{f i n}\left(Y, k_{T}\right)=N_{f i n}(Y, r)=\int d Y_{0} G\left(Y-Y_{0}, r\right) N_{\text {in }}\left(Y_{0}, r=r_{0}\right)
$$

In other word, eq. (2.8) is a realization of the evolution in $r$.

We use the Mellin transform to find $G\left(r, Y-Y_{0}\right)$ in the for

$$
\begin{aligned}
G\left(Y-Y_{0}, r\right) & =\int_{a-i \infty}^{a+i \infty} \frac{d \omega}{2 \pi i} G(\omega, r) e^{\omega\left(Y-Y_{0}\right)} \\
G(\omega, r) & =\int_{a-i \infty}^{a+i \infty} \frac{d f}{2 \pi i} g(\omega, f) \varphi_{f}(r) \\
& =\int_{a-i \infty}^{a+i \infty} \frac{d f}{2 \pi i} g(\omega) e^{\frac{1}{b \omega} \int_{f}^{f_{0}} \chi\left(f^{\prime}\right) d f^{\prime}+r f}
\end{aligned}
$$

where $\chi(f)$ is the Mellin transform of the $K_{L O}$. Solution of eq. (2.10) was firstly written in ref. [7] and has been discussed in details (see refs. [9, 10] and references therein).

In this paper we will proceed with the diffusion approximation for $\chi(f)$ for the sake of simplicity. A generalization is simple and straightforward. Therefore

$$
\chi(f)=\chi_{0}+D_{0}\left(f-\frac{1}{2}\right)^{2} \quad \text { with } \quad \chi_{0}=4 \ln 2=2.772 \text { and } D_{0}=14 \zeta(3)=16.828
$$




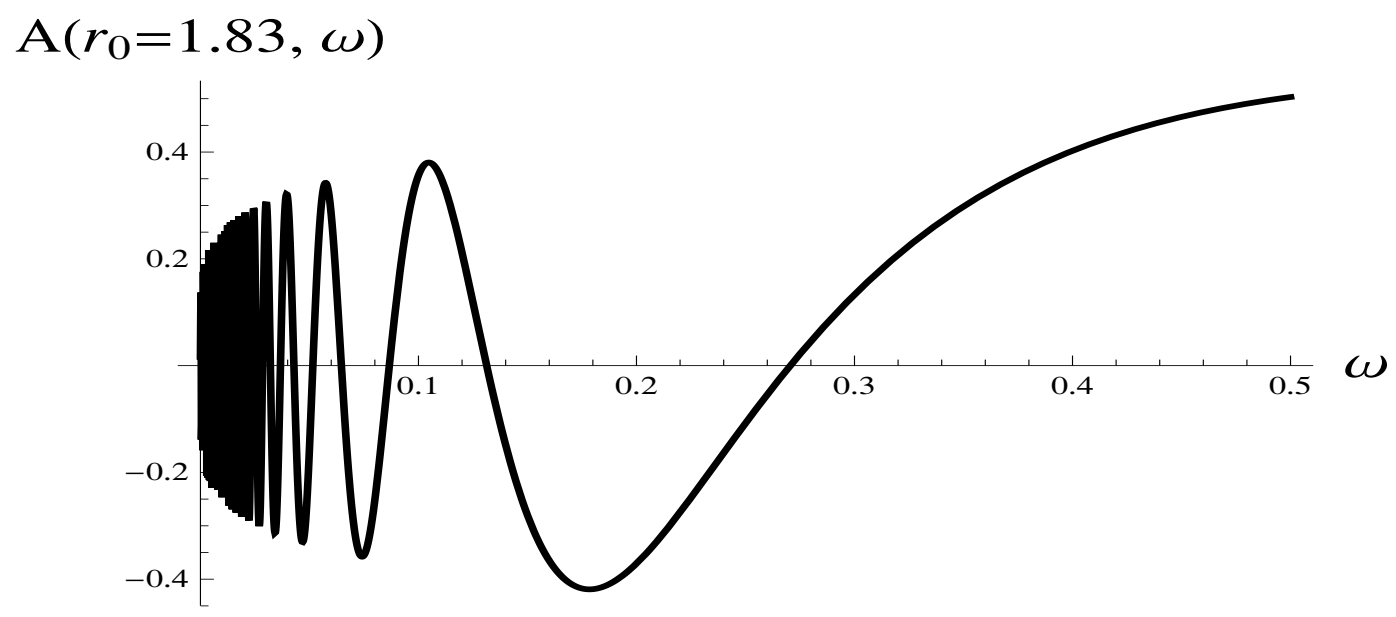

Figure 1. Function $A\left(\omega, r=r_{0}\right)$ versus $\omega . r_{0}=1.83$.

The general solution is

$$
G\left(Y-Y_{0}, r\right)=\int_{a-i \infty}^{a+i \infty} \frac{d \omega}{2 \pi i} \int_{f_{0}-i \infty}^{f_{0}+i \infty} \frac{d f}{2 \pi i} \tilde{g}(\omega) e^{\omega\left(Y-Y_{0}\right)+f r-\frac{\left(\chi_{0} f+\frac{D_{0}}{3} f^{3}\right)}{b \omega}}
$$

Denoting

$$
A(\omega, r)=\int_{f_{0}-i \infty}^{f_{0}+i \infty} \frac{d f}{2 \pi i} e^{f r-\frac{\left(\chi_{0} f+\frac{D_{0}}{3} f^{3}\right)}{b(r) \omega}}
$$

one can see that Green's function which satisfies eq. (2.7) is equal to

$$
G(y, r)=\int_{a-i \infty}^{a+i \infty} \frac{d \omega}{2 \pi i} e^{\omega\left(y-y_{0}\right)} \frac{A(\omega, r)}{A\left(\omega, r_{0}\right)}
$$

For our simplified BFKL kernel

$$
A(\omega, r)=\left(\frac{b \omega}{D_{0}}\right)^{1 / 3} A i\left(\left(r-\frac{\chi_{0}}{b \omega}\right)\left(\frac{b \omega}{D_{0}}\right)^{1 / 3}\right)
$$

One can see that this solution has a discrete spectrum [8] of states that are determined by the zeros of $A\left(\omega, r_{0}\right)$ or by the roots of the following equation

$$
A i\left(\left(r_{0}-\frac{\chi_{0}}{b \omega}\right)\left(\frac{b \omega}{D_{0}}\right)^{1 / 3}\right)=0
$$

In figure 1 it is plotted function $A\left(\omega, r=r_{0}\right)$ versus $\omega$. One can see that we have the set of zeros which condenses to zero.

Airy functions have zeros only at the negative values of the argument, and their position can be found with good accuracy from the simple equation:

$$
z=-\left(\frac{3 \pi n}{2}-\frac{3 \pi}{8}\right)^{\frac{2}{3}}
$$




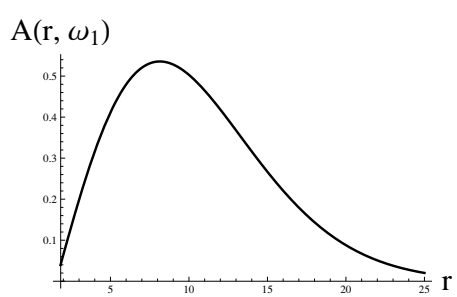

(a)

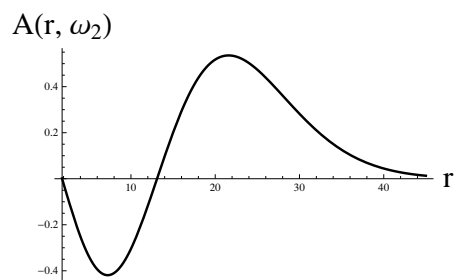

(b)

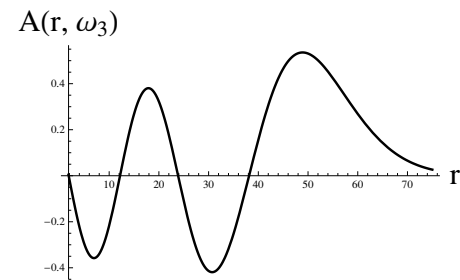

(c)

Figure 2. Function $A\left(\omega_{n}, r\right)$ versus $r . r_{0}=1.83$ and $\omega_{1}=0.27, \omega_{2}=0.1312$ and $\omega_{3}=0.0646$.

Using eq. (2.17) we can find the spectrum of the BFKL equation analytically, solving the equation

$$
\left(\left(r_{0}-\frac{\chi_{0}}{b \omega_{n}}\right)\left(\frac{b \omega_{n}}{D_{0}}\right)^{1 / 3}\right)=-\left(\frac{3 \pi n}{2}-\frac{3 \pi}{8}\right)^{\frac{2}{3}}
$$

At large $n$ we have a solution

$$
\omega_{n}=\frac{2}{3 \pi b n}\left(\frac{\chi_{0}}{D_{0}^{1 / 3}}\right)^{3 / 2}
$$

Finally, the spectrum of the BFKL Pomeron depends only on initial value of $r_{0}=$ $\ln \left(k_{0, \perp}^{2} \Lambda_{\mathrm{QCD}}^{2}\right)$ while the residues depend on the measured $r$. Resulting Green's function is the sum of the infinite number of the Regge poles which residues depend on $r$ (see figure 2) while their positions are determined by $r_{0}$. All features of these poles are the same as in the procedure suggested in refs. [3, 4, 9-12]. The difference of our approach in comparison with the approach of those papers, is in the specific form how we impose the confinement on the BFKL equation. It is well known that the BFKL approach cannot be implemented without introducing the restriction that stem from the confinement region [28]. In figure 3 the typical distribution of the gluon momenta in the BFKL Pomeron is presented. For the values of the transverse momenta $q \leq q_{0}$ the unknown mechanism of confinement of quark and gluons plays the dominant role. We took the following approach to introduce the confinement to the BFKL evolution: we put the initial condition at $q_{\text {in }}=q_{0}\left(N_{\text {in }}\right.$ in eq. (2.8) $)$ and consider the BFKL evolution only for the transverse momenta of partons $\left(k_{\perp} \geq q_{0}\right)$,

This initial condition should be determined from the non-perturbative QCD. The high energy phenomenology [36-40] as well as $\mathrm{N}=4 \mathrm{SYM}[41,42]$ lead to

$$
N_{\mathrm{in}}\left(Y_{0}, r=r_{0}\right)=g_{\mathbb{P}}\left(Y_{0}\right) e^{\Delta_{\mathbb{P}} Y_{0}}+g_{\mathbb{R}}\left(Y_{0}\right) e^{\Delta_{\mathbb{R}} Y_{0}}
$$

where $\Delta_{\mathbb{P}}\left(\Delta_{\mathbb{R}}\right)$ is the Pomeron (secondary Reggeon) intercept, respectively. The physical meaning of the two terms in eq. (2.20) is clear in the high energy phenomenology based on the Reggeon approach. The first contribution describes the contribution of the soft Pomeron and its intercept will be a parameter of our fit. Function $g_{\mathbb{P}}$ is the residue of the Pomeron contribution in which we include also the $\ln Y_{0}$ dependence which can stem from the Pomeron interactions. The second term in eq. (2.20) is responsible for the exchange of 


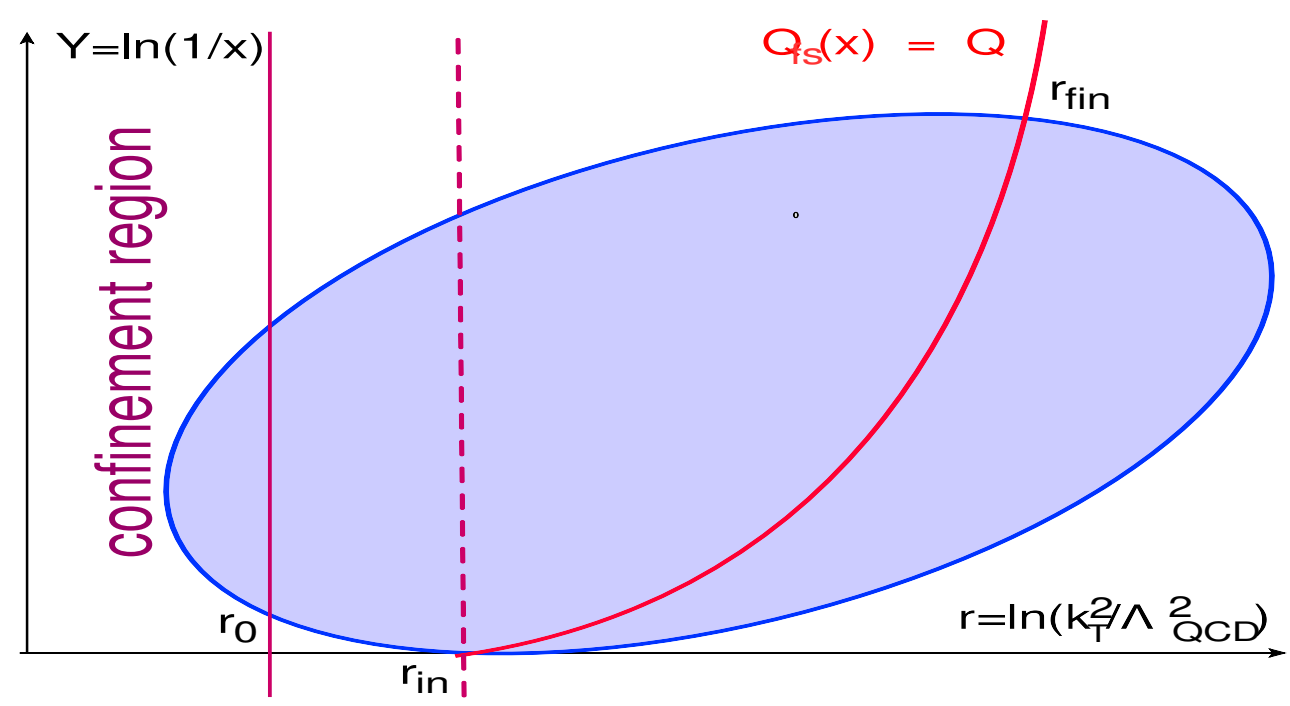

Figure 3. The distribution of the transverse momenta of gluon in the BFKL Pomeron for BFKL evolution from $q^{2}=q_{\text {in }}^{2}\left(r=r_{\text {in }}\right)$ which does not coincide with $r=r_{0}$ (Bartels sigar [28]). The solution to the equation $Q=Q_{s}(x)$ is shown in red. The region to the left of this curve is the saturation region of the non linear evolution. The region to the right of the curve is the region where we can trust the linear BFKL equation. The dashed red line shows the case when $r_{0}=r_{\text {in }}$ which we consider in the paper.
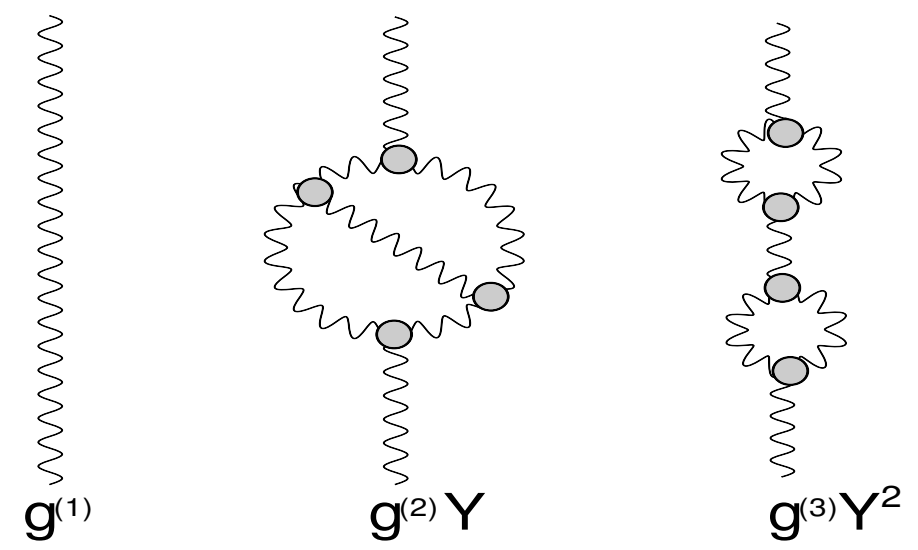

Figure 4. The examples of the Pomeron diagrams that lead to $Y$ and $Y^{2}$ dependence in eq. (2.21). The wave lines denote soft Pomerons.

the secondary Reggeon. We fix the value of $\Delta_{\mathbb{R}}=-0.5$ in our fit. For $g_{\mathbb{P}}\left(Y_{0}\right)$ and $g_{\mathbb{R}}\left(Y_{0}\right)$ we assume the simple form

$$
g_{\mathbb{P}}\left(Y_{0}\right)=g_{\mathbb{P}}^{(1)}+g_{\mathbb{P}}^{(2)} Y_{0}+g_{\mathbb{P}}^{(3)} Y_{0}^{2} ; \quad g_{\mathbb{R}}\left(Y_{0}\right)=g_{\mathbb{R}}^{(1)}+g_{\mathbb{R}}^{(2)} Y_{0}+g_{\mathbb{R}}^{(3)} Y_{0}^{2}
$$

The polynomial in $Y_{0}$ reflects the enhanced diagrams for Pomeron interaction shown in figure 4. 


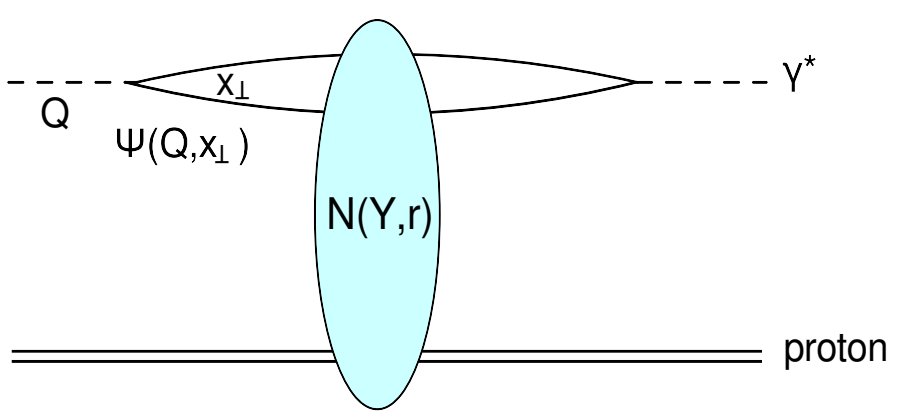

Figure 5. Deep inelastic scattering in dipole approach. Dashed line denotes a photon with virtuality $Q$. The solid lines with arrows describe the quarks and antiquarks. $N\left(Y, r=\ln \left(1 /\left(x_{\perp}^{2} \Lambda_{\mathrm{QCD}}^{2}\right)\right)\right)$ is the dipole-proton scattering amplitude, $Y=\ln \left(1 / x_{B j}\right)$ where $x_{B j}$ is the Bjorken $x$.

\subsection{Main formulae}

In $\omega$-representation eq. (2.19) and eq. (2.21) can be written in the form

$$
g^{\lambda_{i}}(\omega)=\frac{g_{i}^{(1)}}{\omega-\lambda_{I}}+\frac{g_{i}^{(2)}}{\left(\omega-\lambda_{i}\right)^{2}}+\frac{2 g_{i}^{(3)}}{\left(\omega-\lambda_{i}\right)^{3}}
$$

where $\lambda_{1}=\Delta_{\mathbb{P}}$ and $\lambda_{2}=\Delta_{\mathbb{R}}$.

Using eq. (2.14),eq. (2.8),eq. (2.9) and eq. (2.10) we can re-write the dipole-target amplitude:

$$
\begin{aligned}
N(Y, r)= & \sum_{i=1}^{2}\left\{g^{\left(\lambda_{i}\right)} R\left(\omega=\lambda_{i} ; r, r_{0}\right)+\left.g_{i}^{(2)} \frac{d R\left(\omega=\lambda_{i} ; r, r_{0}\right)}{d \omega}\right|_{\omega=\lambda_{i}}\right. \\
& \left.+\left.g_{i}^{(3)} \frac{d^{2} R\left(\omega=\lambda_{i} ; r, r_{0}\right)}{d \omega^{2}}\right|_{\omega=\lambda_{i}}\right\} e^{\lambda_{i} Y} \\
& +\sum_{i=1}^{2} \sum_{n=1}^{\infty} \frac{g^{\lambda_{i}}\left(\omega_{n}\right)}{\omega_{n}-\lambda_{i}} e^{\omega_{n} Y} \frac{A i\left(\left(r-\frac{\chi_{0}}{b \omega_{n}}\right)\left(\frac{b \omega_{n}}{D_{0}}\right)^{1 / 3}\right)}{A i_{\omega=\omega_{n}}^{\prime}}
\end{aligned}
$$

where

$$
\begin{aligned}
R\left(\omega, r, r_{0}\right)= & \frac{A i\left(\left(r-\frac{\chi_{0}}{b \omega}\right)\left(\frac{b \omega}{D_{0}}\right)^{1 / 3}\right)}{A i\left(\left(r_{0}-\frac{\chi_{0}}{b \omega}\right)\left(\frac{b \omega}{D_{0}}\right)^{1 / 3}\right)} \\
& \text { and } A i\left(\left(r_{0}-\frac{\chi_{0}}{b \omega}\right)\left(\frac{b \omega}{D_{0}}\right)^{1 / 3}\right) \stackrel{\omega \rightarrow \omega_{n}}{\longrightarrow} A i_{\omega=\omega_{n}}^{\prime}\left(\omega-\omega_{n}\right)
\end{aligned}
$$

All above formulae have been written in the momentum representation. For calculating $F_{2}(Q, Y)$ it is more convenient to use the coordinate representation going from the dipole transverse momentum to the size of the dipole. Such a transformation it is easy to do in eq. (2.23) by just replacing $r=\ln \left(k_{\perp}^{2} / \Lambda_{\mathrm{QCD}}^{2}\right) \rightarrow r=\ln \left(1 /\left(x_{\perp}^{2} \Lambda_{\mathrm{QCD}}^{2}\right)\right)$ where $x_{\perp}$ is the dipole size. 
For calculating the amplitude for the deep inelastic scattering we need to recall that this process happens through the virtual photon fluctuating into a $q \bar{q}$ pair(dipole) with the $q \bar{q}$ pair proceeding to interact with the target [29-31]. The cross section for the DIS process in this dipole picture can be written as follows [32-35]

$$
\begin{aligned}
\sigma_{\text {tot }}^{T, L}\left(\gamma^{*}+\operatorname{proton} \mid Y\right. & \left.=\ln \left(1 / x_{B j}\right), Q^{2}\right) \\
& =\int \frac{d^{2} x_{\perp}}{4 \pi} \int_{0}^{1} \frac{d z}{z(1-z)}\left|\Psi_{T, L}^{\gamma^{*} \rightarrow q \bar{q}}\left(\vec{x}_{\perp}, z\right)\right|^{2} \sigma_{\text {tot }}^{q \bar{q}, \operatorname{proton}}\left(\vec{x}_{\perp}, Y\right) .
\end{aligned}
$$

where $\left|\Psi_{T, L}\right|^{2}$ is the probability to find the dipole with size $x_{\perp}$ into virtualphoton with transverse or longitudinal polarization; and $\sigma_{\text {tot }}$ is the total cross section of $q \bar{q}$ (dipole) interaction with the proton. The wave function of the virtual photon are known [30, 35]

$$
\begin{aligned}
&\left|\Psi_{T}^{\gamma^{*} \rightarrow q \bar{q}}\left(\vec{x}_{\perp}, z\right)\right|^{2}=2 N_{c} \sum_{f} \frac{\alpha_{\mathrm{em}} Z_{f}^{2}}{\pi} z(1-z) \times \\
& \times\left\{a_{f}^{2}\left[K_{1}\left(x_{\perp} a_{f}\right)\right]^{2}\left[z^{2}+(1-z)^{2}\right]+m_{f}^{2}\left[K_{0}\left(x_{\perp} a_{f}\right)\right]^{2}\right\} \\
&\left|\Psi_{L}^{\gamma^{*} \rightarrow q \bar{q}}\left(\vec{x}_{\perp}, z\right)\right|^{2}=2 N_{c} \sum_{f} \frac{\alpha_{\mathrm{em}} Z_{f}^{2}}{\pi} 4 Q^{2} z^{3}(1-z)^{3}\left[K_{0}\left(x_{\perp} a_{f}\right)\right]^{2} .
\end{aligned}
$$

where

$$
a_{f}^{2}=z(1-z) Q^{2}+m_{f}^{2},
$$

$\alpha_{\mathrm{em}}$ is the fine-structure constant and $Z_{f}$ is the fraction of the electron charge that carries by the quark(antiquark) with flavour $f$ and mass $m_{f}$.

Finally, we need to recall that

$$
\begin{aligned}
F_{2}\left(x, Q^{2}\right) & =\frac{Q^{2}}{4 \pi^{2} \alpha_{\mathrm{em}}} \sigma_{\mathrm{tot}}\left(\gamma^{*}+\text { proton }\right) \\
& =\frac{Q^{2}}{4 \pi^{2} \alpha_{\mathrm{em}}}\left[\sigma_{\mathrm{tot}}^{T}\left(\gamma^{*}+\text { proton }\right)+\sigma_{\mathrm{tot}}^{L}\left(\gamma^{*}+\text { proton }\right)\right]
\end{aligned}
$$

\section{Description of the HERA data}

Using formulae of the previous subsection we describe the HERA data on the deep inelastic structure function $F_{2}$. This set of data was published in ref. [20] and presents the combined data set of ZEUS and H1 collaborations. The experimental errors are small and to describe these data is a challenge for any theoretical approach. In our procedure of the description we see two sets of the phenomenological parameters: the intercept of the soft Pomeron $\lambda_{1}$ and two functions $g_{\mathbb{P}}\left(Y_{0}\right)$ and $g_{\text {reg }}\left(Y_{0}\right)$, which are characterized the initial non-perturbative function of $x_{B j}\left(Y=\ln \left(1 / x_{B j}\right)\right)$ at $Q^{2}=Q_{0}^{2}\left(r=r_{0}\right)$; and two inputs for the $Q^{2}$ evolution: the initial val;ue of $Q=Q_{0}$ from which we start the evolution in $\ln \left(Q^{2}\right)\left(Q>Q_{0}\right)$ and the mass of the quarks $\left(m_{f}\right)$. It turns out that the value of $m_{f}$ in all our fits $\leq 10 \mathrm{MeV}$ and, therefore, we are dealing with current quarks as it should be in our approach. For 
simplicity we use the same masses $m_{f}$ for all light quarks. The substitutions of the current mass from the PGD table: $m_{u}=2.3 \mathrm{MeV}, m_{d}=4.8 \mathrm{MeV}$ and $m_{s}=95 \mathrm{MeV}$, diminish the value $\chi^{2} /$ d.o.f. but only marginally.

As far as the fit of the initial function of $Y_{0}$, it turns out that we have a set of fits with different values of the parameters(see table 1). One can see from this table that we found the set of solutions which have in common the fact that $\lambda_{1}^{(n)}$ are close to the position of the poles in the Green function $\omega_{n}$. The differences between $\lambda_{1}^{(n)}-\omega_{n}$ is so small that parameter $\left(\lambda_{1}^{(n)}-\omega_{n}\right) Y \ll 1$ for the HERA kinematic region. It means that we actually claim that the intercept of the soft Pomeron coincides with the one of poles that appears in the Green function. In this situation we need to rewrite eq. (2.23) selecting separately the contribution with $\lambda_{1}^{(k)}=\omega_{k}$ : viz

$$
\begin{aligned}
N(Y, r)= & \left\{g_{1}^{(1)} Y+\frac{1}{2} g_{1}^{(2)} Y^{2}+\frac{1}{3} g_{1}^{(3)} Y^{3}\right\} \frac{A i\left(\left(r-\frac{\chi_{0}}{b \omega_{k}}\right)\left(\frac{b \omega_{k}}{D_{0}}\right)^{1 / 3}\right)}{A i_{\omega=\omega_{k}}^{\prime}} e^{\omega_{k} Y} \\
& +\left\{g^{\left(\lambda_{2}\right)} R\left(\omega=\lambda_{2} ; r, r_{0}\right)+\left.g_{2}^{(2)} \frac{d R\left(\omega=\lambda_{2} ; r, r_{0}\right)}{d \omega}\right|_{\omega=\lambda_{2}}\right. \\
& \left.+\left.g_{2}^{(3)} \frac{d^{2} R\left(\omega=\lambda_{2} ; r, r_{0}\right)}{d \omega^{2}}\right|_{\omega=\lambda_{2}}\right\} e^{\lambda_{2} Y} \\
& +\sum_{i=1}^{2} \sum_{n=1}^{\infty} \frac{g^{\lambda_{i}}\left(\omega_{n}\right)}{\omega_{n}-\lambda_{i}} e^{\omega_{n} Y} \frac{A i\left(\left(r-\frac{\chi_{0}}{b \omega_{n}}\right)\left(\frac{b \omega_{n}}{D_{0}}\right)^{1 / 3}\right)}{A i_{\omega=\omega_{n}}^{\prime}}
\end{aligned}
$$

where $\sum^{\prime}$ denotes the sum without the term with $n=k$. It should be stressed that in spite of the fact that the largest contribution stems from one term in sum in eq. (2.23), we have to sum up to $n=N_{\max } \approx 200$ to obtain the accuracy of our calculation smaller than the experimental errors. All these solutions lead to good $\chi^{2} /$ d.o.f and the reason why we have them is clear from figure $6(\mathrm{a})$ in which we plotted the values of $N_{\text {in }}\left(Y_{0}, r_{0}\right)$ in eq. (2.8). One can see that in the HERA kinematic range ( to the left from the vertical line in figure 6(a)) all solutions give the same $N_{\text {in }}$ and the difference started to be visible only for larger values of $Y_{0}$.

It should be stressed that our initial condition cannot be describe by the contribution of only two Regge poles: Pomeron and the secondary trajectory. We need to take into account the interaction of the Pomerons. On the other hand in our parameterization we restrict ourselves by contribution of the enhanced diagrams (see figure 4), In other words it looks that we do not need to take into account the screening corrections. First of all it is very good that we do not need the screening correction in the initial condition: this makes our approach self-consistent since we do not take into account the non-linear corrections in the evolution. The fact that we can describe the HERA experimental data without non-linear corrections is well known. It is enough to mention that CTEQ [13-15],MRST [16-18], HERAPDF [20] parameterizations as well as a dozen of others in Durham HEP data, give the description based on linear evolution. The attempts to fit HERA data in $\mathrm{N}=4$ SYM also showed that we do not need to take into account non-linear contributions [43, 


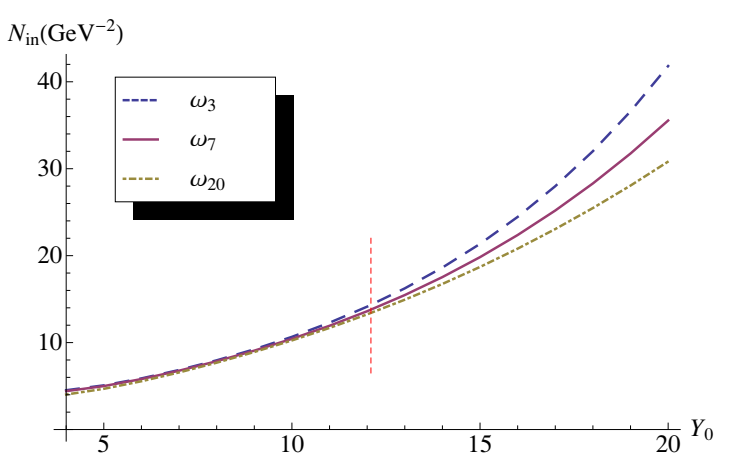

(a)

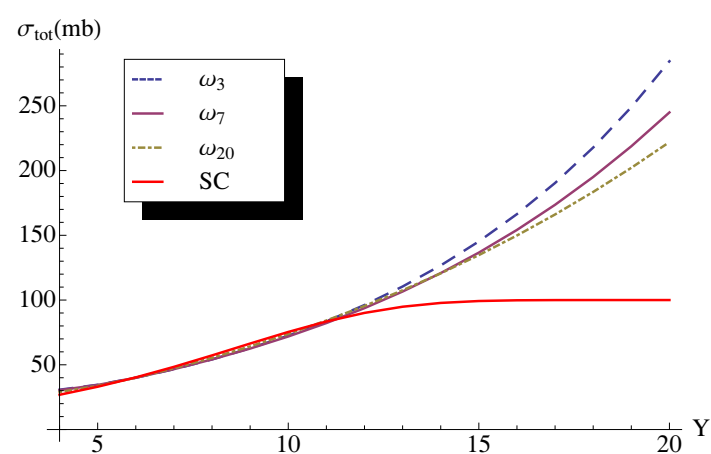

(b)

Figure 6. Figure 6(a): $N\left(Y_{0}, k_{0}\right)=N_{\text {in }}\left(Y_{0}, r_{0}\right)$ defined in eq. (2.2) versus $Y_{0}$ for different solutions in our fit. $\lambda_{1}^{(n)}$ are shown in the legend. The vertical line shows the maximal value of $Y_{0}$ in HERA experiment which we took into account in our fit. Figure 6(b): the same function $N_{\text {in }}\left(Y_{0}, r_{0}\right)$ as in figure 6(a) but normalized to the value of the total cross section for proton-proton interaction at $W=20 \mathrm{GeV} . Y=\ln \left(s / s_{0}\right)$ with $s_{0}=1 \mathrm{GeV}^{2}$. The red solid curve gives eq. (3.2) in the text with $\sigma_{0}=100 \mathrm{mb}, \kappa=0.115$ and $\Delta=0.25$.

44]. On the other hand, we are aware that the data can be described in the saturation models with substantial non-linear corrections ( see refs. [45-48]). We need to comment on ref. [48] in which the most theoretical approach is suggested: to solve non-linear BalitskyKovchegov (BK) equation [49-51] with running QCD coupling. First, in this paper the triumvirate structure of eq. (2.5) is used for accounting of the running $\alpha_{S}$ while we used eq. (2.6). Second, in ref. [48] the additional assumption is made: freezing of the running QCD coupling which does not follow from BK equations, and the third, it is assumed that the impact parameter dependence of the initial condition (at fixed $Y=Y_{0}$ ) is $\Theta(R-b)$, where $R$ is the proton radius that does not depend on energy. Certainly, such assumption contradicts all experimental data on proton but it also does not reduce the BK equation to the equation without $b$ dependence, which was solved in this paper, since for this purpose we need to assume such kind of $\Theta$ function for the BFKL kernel (see ref. [52]).

Concluding this discussion we believe that we present the theoretically self-consistent description of the HERA data but we accept the fact that the HERA data could be described in a different way as well.

However, our approach has to pass another check on self-consistency. Indeed, having the exchange of the soft Pomeron as the initial condition, we need to check that this soft Pomeron does not contradict the data on proton-proton cross section at high energy where the shadowing corrections should be taken into account. The contribution of the soft Pomeron to the scattering amplitude of the proton-proton interaction differs from the contribution to the initial condition in DIS by the constant factor which we fix by the condition that $\sigma_{\text {tot }}$ (proton-proton) $=40 \mathrm{mb}$ at $W=20 \mathrm{GeV}$. We found that the simple formula with screening corrections:

$$
\sigma_{\text {tot }}=\sigma_{0}\left(1-\exp \left(-\kappa e^{\Delta Y}\right)\right)
$$

is able to describe the initial condition in the HERA kinematic region and leads to qualita- 
tively reasonable values of the total cross sections at large $Y$ (see figure 6(b)). It worthwhile mentioning that $\Delta$ that gives the description, is rather large $(\Delta=0.25)$ in agreement with the recent outcome from high energy Regge phenomenology [36-40]. Eq. (3.2) is oversimplified but correctly introduce three main parameters of the soft Pomeron: intercept $\Delta$, vertex of Pomeron-proton interaction $(\sqrt{\kappa})$ and the size of the Pomeron in $b$-space $\sigma_{0}$. Figure 6 demonstrates that the initial conditions without shadowing corrections in the HERA kinematic region passes the check on self-consistency: they do not contradict the behaviour of the total proton-proton cross sections at higher energy where the shadowing correction should be taken into account.

To complete discussion on the role of the non-linear corrections we need the estimate the contribution of the non-linear term in the evolution equation in the HERA kinematic region using our solution. The easiest way is to find $N\left(x_{\perp}, b ; Y\right)$ and compare it with unity. However, the DIS data give us information on the dipole scattering amplitude integrated over $b$. Therefore, we have to replace $N\left(x_{\perp}, b ; Y\right)$ by $\left\langle N\left(x_{\perp} ; Y\right)\right\rangle=$ $\int d^{2} b N\left(x_{\perp}, b ; Y\right) / \pi R^{2}$ where $R$ is the size of the proton. This formula, which can be rather good for the DGLAP solution, is certainly very approximate for the solution of the BFKL equation since the radius of proton rapidly increases with energy [53]. Substituting the smallest radius of proton which exists on the market $\left(R^{2}=8 \div 10 \mathrm{GeV}^{-2}\right.$ see refs. [45$\left.47]^{1}\right)$ we found that $\int d^{2} b N\left(x_{\perp}, b ; Y\right) / \pi R^{2} \leq 0.05 \div 0.1$ in the entire HERA kinematic region. Such corrections we can neglect within $\chi^{2} /$ d.o.f. that we obtained, especially because, we overestimated the values of $N\left(x_{\perp}, b ; Y\right)$ using so small radius. This fact is clear when we consider the value of $N\left(x_{\perp}, b ; Y\right)$ for the value of $x_{\perp}$ in initial conditions (see figure 6(a)). At first sight using $R^{2}=8 \div 10 \mathrm{GeV}^{-2}$ we obtain that $N\left(x_{\perp}, b ; Y\right) \approx 0.3<1$ at $Y=12$. Certainly it is not true since for the initial condition we need to use larger radius, for example, the value of the elastic slope of $\pi$-proton scattering at $Y=12$. Such estimate gives $R^{2}=30 \div 35 \mathrm{GeV}^{-2}$ leading to $N\left(x_{\perp}, b ; Y\right)=\int d^{2} b N\left(x_{\perp}, b ; Y\right) / \pi R^{2} \leq 0.1$. The values larger than $N_{\text {in }}\left(Y_{0}\right)=13$ are outside of the HERA kinematic region and we believe that the shadowing correction should be taken into account at such large $Y$ as we illustrate in figure 6(b). However, it is interesting to note that the maximum value $N_{\text {in }}\left(Y_{0}\right)=40$ in figure 6 (a) still leads to $\int d^{2} b N\left(x_{\perp}, b ; Y_{0}\right) / \pi R^{2} \approx 0.3<1$ with $R^{2}=30 \div 35 \mathrm{GeV}^{-2}$.

The natural question arises, whether we can use the linear BFKL equation and take into account the enhanced diagrams of figure 4 in the initial conditions. The contribution of the first enhanced diagram in the BFKL Poimeron ecxchange is proportional to $\bar{\alpha}_{S}^{3} / N_{c}^{2} Y$ and for small values of the running QCD coupling these corrections are neglected since in the leading $\log (1 / \mathrm{x})$ approximation we account for the corrections of the order of $\left(\bar{\alpha}_{S} Y\right)^{n}$. However, in the non-perturbative region the enhanced diagram are the first that have to be included since they give contribution to the intercept of the soft Pomeron. It should be noticed that the parameter that is reponsible for the size of these corrections: $\left(g_{\mathbb{P}}^{(2)} / g_{\mathbb{P}}^{(2)}\right) Y \leq 0.07 Y$ (see table 1 ) is rather small in accordance with the perturbative QCD estimates.

\footnotetext{
${ }^{1}$ We introduce $R^{2}=2 B$ where $B$ is the slope of the Gaussian impact parameter form factor $T(b)=$ $(1 / 2 \pi B) \exp \left(-b^{2} / 2 B\right)$.
} 


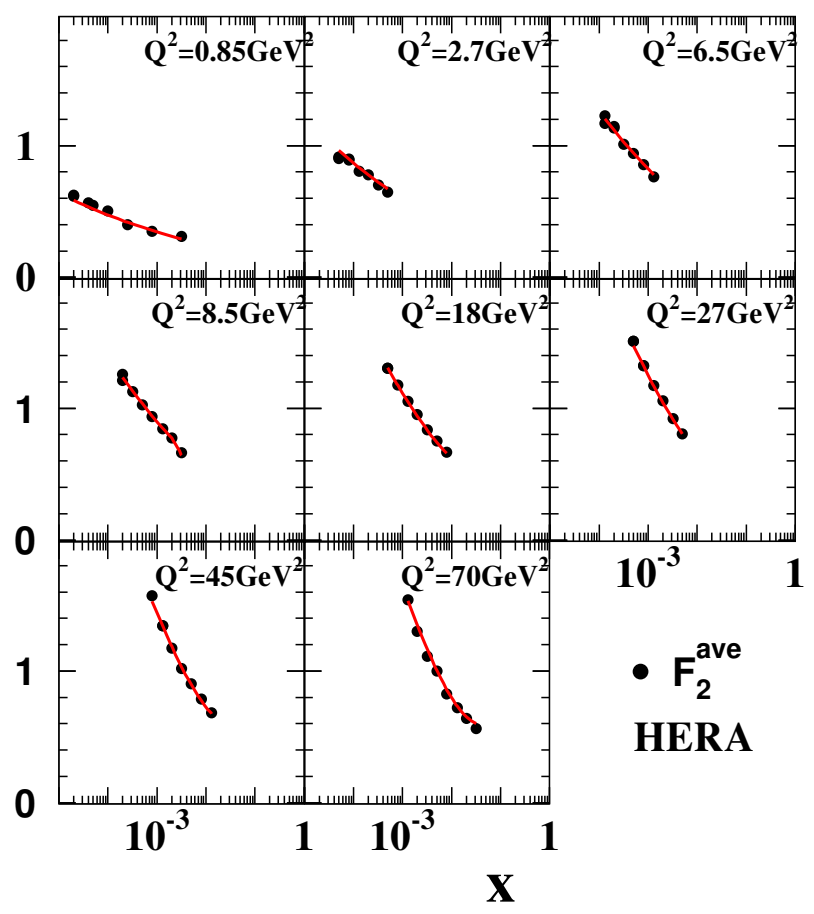

Figure 7. The deep inelastic structure function $F_{2}$ versus $x$. The data are taken from ref. [20]. $r_{0}=1.83$. All other parameters in table 2 for $\lambda_{1}^{(7)}$.

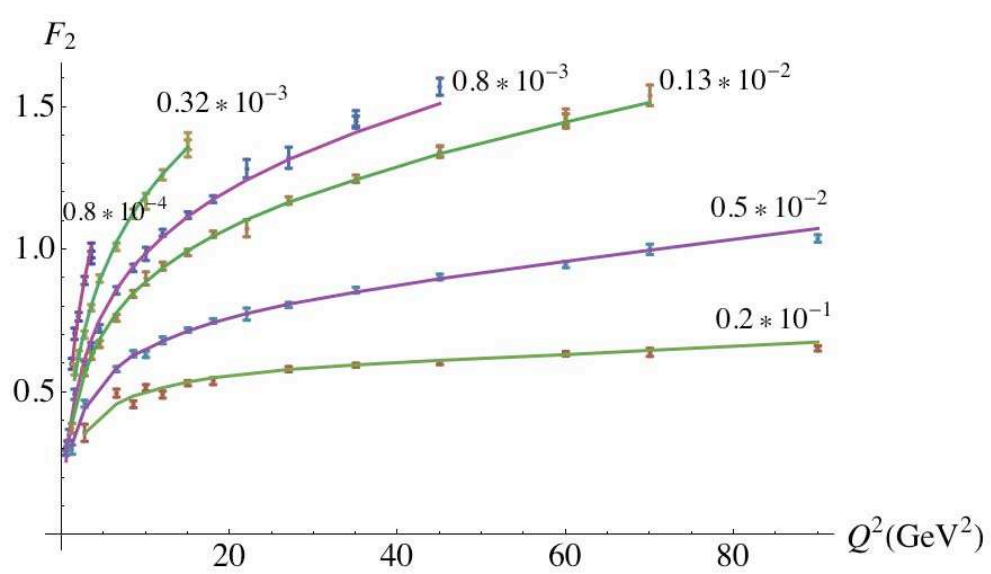

Figure 8. The deep inelastic structure function $F_{2}$ versus $Q$ at fixed $x_{B j}$. The data are taken from ref. [20]. $r_{0}=1.83$. All other parameters in table 1 for $\lambda_{1}^{(7)}=\omega_{7}$. The values of $x_{B j}$ are shown in the figure.

Our main fitting parameter that is responsible for $Q^{2}$ evolution is $r_{0}$. We found that the best $\chi^{2} /$ d.o.f. we obtain for $q_{0}^{2}=0.25 \mathrm{GeV}^{2}\left(r_{0}=1.83\right)$ for any choice of $\lambda^{(k)}=\omega_{k}$. However, the minimum of $\chi^{2} /$ d.o.f. is rather shallow. The best $\chi^{2} /$ d.o.f. we found for $\lambda^{(k)}=\omega_{k}=\omega_{7}$ (see table 1$)$.

The quality of the fit one can see from figure 7 and figure 8.

Different solutions give the same descriptions: see figure 9 in which we compare the solution with $\lambda_{1}^{(3)}$ and $\lambda_{1}^{(7)}$. 
In figure 10 we plot the calculated value of $d \ln F_{2}\left(x_{B j}, Q^{2}\right) / d \ln \left(1 / x_{B j}\right)$ at different values of $x_{B j}$. The solid lines corresponds to the kinematic region in which we fit the data. The dashed curves can be considered as predictions. One can see that we predict the dependence of this observable on $x_{B j}$ but this dependence is rather mild in the HERA kinematic region.

\section{Conclusions}

In this paper we developed approach based on the BFKL evolution in $\ln \left(Q^{2}\right)$. We show that the simplest diffusion approximation with running QCD coupling is able to describe the HERA experimental data on the deep inelastic structure function with good $\chi^{2} /$ d.o.f. $\approx 1.3$. We consider this result as the strong argument against the wide spread opinion that the BFKL dynamics has not been seen experimentally at HERA. This result confirms the outcome of refs. [9-12], in which the BFKL equation was considered as the theory of the reggeons.

From our description of the experimental data we learned several lessons:

- The non-perturbative physics at long distances started to show up at $Q^{2}=$ $0.25 \mathrm{GeV}^{2}$;

- The scattering amplitude at $Q^{2}=0.25 \mathrm{GeV}^{2}$ cannot be written as sum of soft Pomeron and the secondary Reggeon but the Pomeron interactions should be taken into account;

- The Pomeron interactions can be reduced to the enhanced diagrams and, therefore, we do not see any needs for the shadowing corrections at HERA energies;

- We demonstrated that the shadowing correction could be sizable at higher than HERA energies without any contradiction with our initial conditions.

We believe that these lessons as well as the fact that we can reach a good description of the HERA data in the framework of the BFKL dynamics, can be useful for future attempts to understand the interface between long (soft) and short(hard) distance physics.

\section{Acknowledgments}

We thank our colleagues at UTFSM and Tel Aviv university for encouraging discussions. Our special thanks goes to Clara Salas who shared with us the results of refs. [54-56] before publication. This research was supported by the Fondecyt (Chile) grants 1100648 and 1130549 .

Open Access. This article is distributed under the terms of the Creative Commons Attribution License (CC-BY 4.0), which permits any use, distribution and reproduction in any medium, provided the original author(s) and source are credited. 


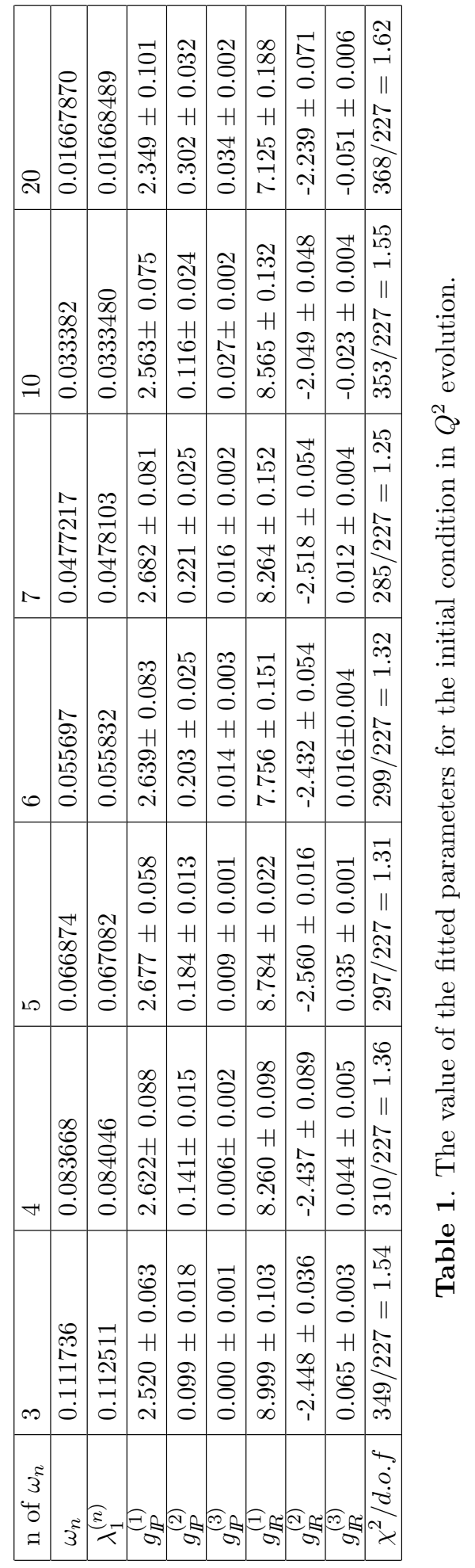




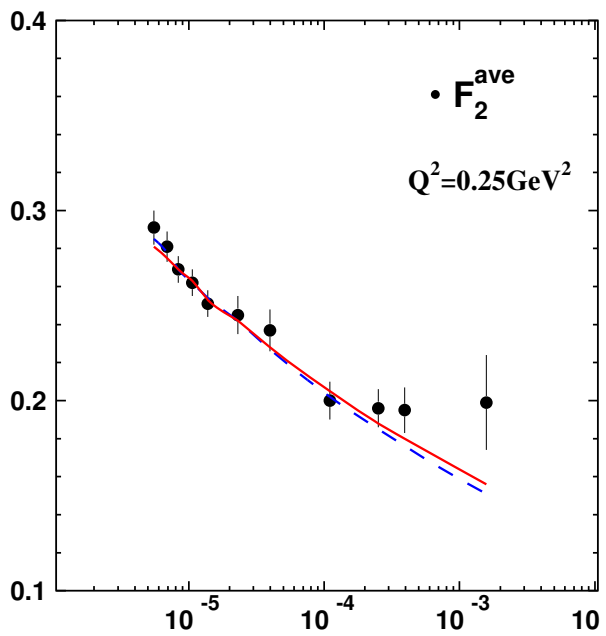

(a)

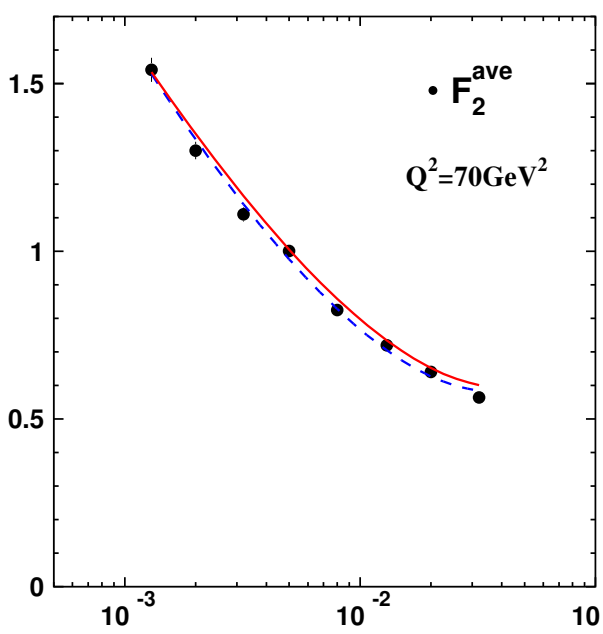

(b)

Figure 9. Comparison of two solutions in our fit with $\lambda_{1}^{(3)}$ (dashed line) and $\lambda_{1}^{(7)}$ (solid line).

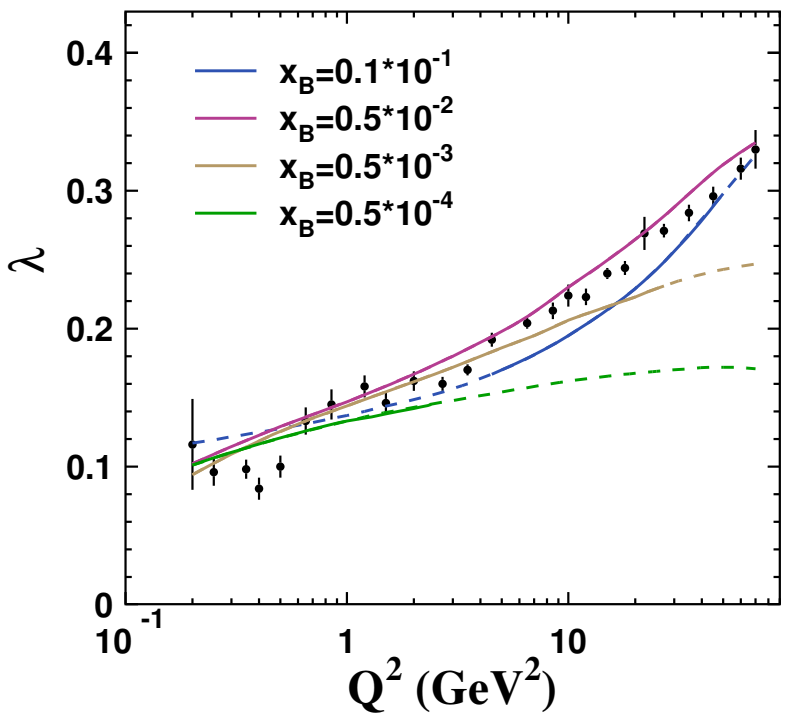

Figure 10. $d \ln F_{2}\left(x_{B j}, Q^{2}\right) / d \ln \left(1 / x_{B j}\right)$ versus $Q^{2}$ at different values of $x_{B j}$ which are shown in the figure. The solid curves describe $d \ln F_{2}\left(x_{B j}, Q^{2}\right) / d \ln \left(1 / x_{B j}\right)$ in the kinematic region of HERA experiment while the dashed curve correspond to the kinematic region outside the HERA region and can be viewed as the predictions. The data points shown in this figure were extracted from the experimental data of ref. [20] by Clara Salas (see refs. [54-56]).

\section{References}

[1] E. Kuraev, L. Lipatov and V.S. Fadin, The Pomeranchuk singularity in nonabelian gauge theories, Sov. Phys. JETP 45 (1977) 199 [INSPIRE].

[2] Ya.Ya. Balitsky and L.N. Lipatov, The Pomeranchuk singularity in quantum chromodynamics, Sov. J. Nucl. Phys. 28 (1978) 822 [Yad. Fiz. 28 (1978) 1597]. 
[3] L. Lipatov, Small $x$ physics in perturbative QCD, Phys. Rept. 286 (1997) 131 [hep-ph/9610276] [INSPIRE].

[4] L. Lipatov, The bare Pomeron in quantum chromodynamics, Sov. Phys. JETP 63 (1986) 904 [INSPIRE].

[5] Y.V. Kovchegov and E. Levin, Quantum choromodynamics at high energies, Cambridge Monographs on Particle Physics, Nuclear Physics and Cosmology, Cambridge University Press, Cambridge U.K. (2012).

[6] H. Abramowicz and A. Caldwell, HERA collider physics, Rev. Mod. Phys. 71 (1999) 1275 [hep-ex/9903037] [INSPIRE].

[7] L. Gribov, E. Levin and M. Ryskin, Semihard processes in QCD, Phys. Rept. 100 (1983) 1 [INSPIRE].

[8] E. Levin, The BFKL high-energy asymptotics in the next-to-leading approximation, Nucl. Phys. B 545 (1999) 481 [hep-ph/9806228] [INSPIRE].

[9] H. Kowalski, L. Lipatov and D. Ross, BFKL evolution as a communicator between small and large energy scales, Phys. Part. Nucl. 44 (2013) 547 [arXiv: 1205.6713] [inSPIRE].

[10] H. Kowalski, L. Lipatov and D. Ross, Indirect evidence for new physics at the $10 \mathrm{TeV}$ scale, arXiv:1109.0432 [INSPIRE].

[11] H. Kowalski, L. Lipatov, D. Ross and G. Watt, The new HERA data and the determination of the infrared behaviour of the BFKL amplitude, Nucl. Phys. A 854 (2011) 45 [InSPIRE].

[12] H. Kowalski, L. Lipatov, D. Ross and G. Watt, Using HERA data to determine the infrared behaviour of the BFKL amplitude, Eur. Phys. J. C 70 (2010) 983 [arXiv:1005.0355] [INSPIRE].

[13] J.F. Owens, A. Accardi and W. Melnitchouk, Global parton distributions with nuclear and finite- $Q^{2}$ corrections, Phys. Rev. D 87 (2013) 094012 [arXiv:1212.1702] [INSPIRE].

[14] J. Gao et al., The CT10 NNLO global analysis of QCD, arXiv:1302.6246 [INSPIRE].

[15] J. Pumplin et al., New generation of parton distributions with uncertainties from global QCD analysis, JHEP 07 (2002) 012 [hep-ph/0201195] [INSPIRE].

[16] A. Martin, W. Stirling, R. Thorne and G. Watt, Heavy-quark mass dependence in global PDF analyses and 3- and 4-flavour parton distributions, Eur. Phys. J. C 70 (2010) 51 [arXiv: 1007.2624] [INSPIRE].

[17] A. Martin, W. Stirling, R. Thorne and G. Watt, Parton distributions for the LHC, Eur. Phys. J. C 63 (2009) 189 [arXiv:0901.0002] [InSPIRE].

[18] A. Martin, W. Stirling, R. Thorne and G. Watt, Update of parton distributions at NNLO, Phys. Lett. B 652 (2007) 292 [arXiv:0706.0459] [INSPIRE].

[19] S. Alekhin, J. Blumlein, S. Klein and S. Moch, Variable-flavor-number scheme in analysis of heavy-quark electro-production data, arXiv:0908.3128 [INSPIRE].

[20] H1 AND ZEUS collaboration, F. Aaron et al., Combined measurement and QCD analysis of the inclusive $e^{ \pm} p$ scattering cross sections at HERA, JHEP 01 (2010) 109 [arXiv:0911.0884] [INSPIRE].

[21] V.S. Fadin and L. Lipatov, BFKL Pomeron in the next-to-leading approximation, Phys. Lett. B 429 (1998) 127 [hep-ph/9802290] [INSPIRE]. 
[22] M. Ciafaloni and G. Camici, Energy scale(s) and next-to-leading BFKL equation, Phys. Lett. B 430 (1998) 349 [hep-ph/9803389] [INSPIRE].

[23] V.S. Fadin and R. Fiore, The generalized nonforward BFKL equation and the 'bootstrap' condition for the gluon Reggeization in the NLLA, Phys. Lett. B 440 (1998) 359 [hep-ph/9807472] [INSPIRE].

[24] I. Balitsky and G.A. Chirilli, High-energy amplitudes in $N=4 S Y M$ in the next-to-leading order, Phys. Lett. B 687 (2010) 204 [arXiv:0911.5192] [INSPIRE].

[25] Y.V. Kovchegov and H. Weigert, Triumvirate of running couplings in small-x evolution, Nucl. Phys. A 784 (2007) 188 [hep-ph/0609090] [INSPIRE].

[26] E. Levin, Renormalons at low x, Nucl. Phys. B 453 (1995) 303 [hep-ph/9412345] [INSPIRE].

[27] M. Braun, High color Pomerons with a running coupling constant in the Hartree-Fock approximation, Phys. Lett. B 351 (1995) 528 [hep-ph/9412202] [INSPIRE].

[28] J. Bartels, A note on the infrared cutoff dependence of the BFKL Pomeron, J. Phys. G 19 (1993) 1611 [INSPIRE].

[29] V. Gribov, Interaction of gamma quanta and electrons with nuclei at high-energies, Sov. Phys. JETP 30 (1970) 709 [INSPIRE].

[30] J. Bjorken and J.B. Kogut, Correspondence arguments for high-energy collisions, Phys. Rev. D 8 (1973) 1341 [INSPIRE].

[31] L. Frankfurt and M. Strikman, Hard nuclear processes and microscopic nuclear structure, Phys. Rept. 160 (1988) 235 [INSPIRE].

[32] B. Kopeliovich, L. Lapidus and A. Zamolodchikov, Dynamics of color in hadron diffraction on nuclei, JETP Lett. 33 (1981) 595 [INSPIRE].

[33] G. Bertsch, S.J. Brodsky, A. Goldhaber and J. Gunion, Diffractive excitation in QCD, Phys. Rev. Lett. 47 (1981) 297 [INSPIRE].

[34] A.H. Mueller, Small x behavior and parton saturation: a QCD model, Nucl. Phys. B 335 (1990) 115 [INSPIRE].

[35] N.N. Nikolaev and B. Zakharov, Color transparency and scaling properties of nuclear shadowing in deep inelastic scattering, Z. Phys. C 49 (1991) 607 [InSPIRE].

[36] E. Gotsman, E. Levin and U. Maor, $N=4 S Y M$ and $Q C D$ motivated approach to soft interactions at high energies, Eur. Phys. J. C 71 (2011) 1553 [arXiv:1010.5323] [INSPIRE].

[37] A.B. Kaidalov and M.G. Poghosyan, Description of soft diffraction in the framework of reggeon calculus: Predictions for $L H C$, arXiv:0909.5156 [INSPIRE].

[38] A. Martin, M. Ryskin and V. Khoze, From hard to soft high-energy pp interactions, arXiv:1110.1973 [INSPIRE].

[39] M. Ryskin, A. Martin and V. Khoze, High-energy strong interactions: from 'hard' to 'soft', Eur. Phys. J. C 71 (2011) 1617 [arXiv:1102.2844] [INSPIRE].

[40] S. Ostapchenko, Monte Carlo treatment of hadronic interactions in enhanced Pomeron scheme: I. QGSJET-II model, Phys. Rev. D 83 (2011) 014018 [arXiv:1010.1869] [INSPIRE].

[41] A. Kotikov, L. Lipatov, A. Onishchenko and V. Velizhanin, Three loop universal anomalous dimension of the Wilson operators in N=4 SUSY Yang-Mills model, Phys. Lett. B 595 (2004) 521 [Erratum ibid. B 632 (2006) 754-756] [hep-th/0404092] [INSPIRE]. 
[42] R.C. Brower, J. Polchinski, M.J. Strassler and C.-I. Tan, The Pomeron and gauge/string duality, JHEP 12 (2007) 005 [hep-th/0603115] [INSPIRE].

[43] E. Levin and I. Potashnikova, Inelastic processes in DIS and N=4 SYM, JHEP 08 (2010) 112 [arXiv: 1007.0306] [INSPIRE].

[44] R.C. Brower, M. Djuric, I. Sarcevic and C.-I. Tan, String-gauge dual description of deep inelastic scattering at small-x, JHEP 11 (2010) 051 [arXiv:1007.2259] [INSPIRE].

[45] A.H. Rezaeian, M. Siddikov, M. Van de Klundert and R. Venugopalan, IP-Sat: Impact-Parameter dependent SATuration model revised, PoS(DIS 2013) 060 [arXiv: 1307.0165] [INSPIRE].

[46] G. Watt and H. Kowalski, Impact parameter dependent colour glass condensate dipole model, Phys. Rev. D 78 (2008) 014016 [arXiv:0712.2670] [INSPIRE].

[47] H. Kowalski, L. Motyka and G. Watt, Exclusive diffractive processes at HERA within the dipole picture, Phys. Rev. D 74 (2006) 074016 [hep-ph/0606272] [INSPIRE].

[48] J.L. Albacete et al., AAMQS: a non-linear QCD analysis of new HERA data at small-x including heavy quarks, Eur. Phys. J. C 71 (2011) 1705 [arXiv: 1012.4408] [INSPIRE].

[49] I. Balitsky, Operator expansion for high-energy scattering, Nucl. Phys. B 463 (1996) 99 [hep-ph/9509348] [INSPIRE].

[50] I. Balitsky, Factorization and high-energy effective action, Phys. Rev. D 60 (1999) 014020 [hep-ph/9812311] [INSPIRE].

[51] Y.V. Kovchegov, Small $x F_{2}$ structure function of a nucleus including multiple Pomeron exchanges, Phys. Rev. D 60 (1999) 034008 [hep-ph/9901281] [INSPIRE].

[52] E. Levin and S. Tapia, BFKL Pomeron: modeling confinement, JHEP 07 (2013) 183 [arXiv: 1304.8022] [INSPIRE].

[53] A. Kovner and U.A. Wiedemann, Nonlinear QCD evolution: saturation without unitarization, Phys. Rev. D 66 (2002) 051502 [hep-ph/0112140] [INSPIRE].

[54] M. Hentschinski, A. Sabio Vera and C. Salas, Description of $F_{2}$ and $F_{L}$ at small $x$ using a collinearly-improved BFKL resummation, Phys. Rev. D 87 (2013) 076005 [arXiv: 1301.5283] [INSPIRE].

[55] C. Salas, BFKL dynamics and collinear resummation: the case of $F_{2}$ and forward jets, invited talk at Low x 2013 WS, May 30-June 4, Rehovot-Eilat, Israel (2013).

[56] C. Salas, private communication.

[57] H1 collaboration, C. Alexa et al., Elastic and proton-dissociative photoproduction of $J / \psi$ mesons at HERA, Eur. Phys. J. C 73 (2013) 2466 [arXiv:1304.5162] [INSPIRE]. 